\title{
Modelo de simulador de injeção laringoplástica transcervical
}

\author{
Transcervical injection laryngoplasty simulation model
}

Modelo de simulación de laringoplastia de inyenccón transcervical

Amanda Martins Umbelino ${ }^{1 *}$, Jussandra Cardoso Rodrigues ${ }^{1}$, Igor Isamu Couceiro Seto ${ }^{1}$, Vanessa Coutinho Aguiar Gomes ${ }^{1}$, Luigi Ferreira e Silva'.

\begin{abstract}
RESUMO
Objetivo: Propor um modelo de simulador de injeção laringoplástica fabricado através da impressão 3D e outros materiais de baixo custo. Métodos: Trata-se de estudo experimental descritivo. A modelagem das estruturas osteocartilaginosas da laringe foi feita a partir de imagens adquiridas através de radiografia do pescoço nas incidências perfil e anteroposterior de um adulto jovem do sexo masculino. Foi utilizada impressora 3D modelo FDM (Fused Deposition Modeling) com o filamento plástico ácido poliláctico para impressão do arcabouço osteocartilaginoso da laringe. As pregas vocais foram representadas através de dedos de luva. Resultados: O arcabouço laríngeo foi modelado de forma a possuir área de encaixe das pregas vocais, ao nível da glote. A base e a estrutura de suporte foram feitas a partir de placa de fibra de madeira sintética de média densidade, sendo fixado o arcabouço laríngeo nesta através de sistema de ímãs. A simulação se dá através da injeção do material no interior das pregas vocais, sendo observado o aumento de volume destas. Conclusão: Foi observado alto grau de fidelidade na representação anatômica e das propriedades táteis do arcabouço osteocartilaginoso, que somado a alta reprodutibilidade e baixo custo torna possível a integração na prática diária dos serviços de residência como nova metodologia de ensino.
\end{abstract}

Palavras-Chave: Laringe, Treinamento por simulação, Otorrinolaringologia.

\begin{abstract}
Objective: To propose a model of laryngoplastic injection simulator manufactured using 3D printing and other low-cost materials. Methods: This is a descriptive experimental study. The modeling of osteocartilaginous structures of the larynx was made from images acquired through radiography of the neck in the lateral and anteroposterior views of a young adult male. 3D printer model FDM (Fused Deposition Modeling) with polylactic acid plastic filament was used to print the osteocartilagenous structures of the larynx. The vocal folds were represented using glove fingers. Results: The laryngeal framework was modeled in such a way that the vocal folds fit into the glottis. The base and the support structure were made from medium density synthetic wood fiber board; the laryngeal framework being fixed to it through a magnet system. The simulation occurs through the injection of material inside the vocal folds, with an increase in their volume being observed. Conclusion: A high degree of fidelity was observed in the anatomical representation and tactile properties of the osteocartilaginous framework, which, in addition to the high reproducibility and low cost, makes it possible to integrate residency services into the daily practice as a new teaching methodology
\end{abstract}

Keywords: Larynx, Simulation training, Otolaryngology.

\section{RESUMEN}

Objetivo: Proponer un modelo de simulador de inyección laringoplástica fabricado mediante impresión 3D y otros materiales de bajo coste. Métodos: Se trata de un estudio experimental descriptivo. El modelado de las estructuras osteocartilaginosas de la laringe se realizó a partir de imágenes obtenidas mediante radiografía del cuello en las vistas lateral y anteroposterior de un varón adulto joven. Se utilizó el modelo de impresora 3D FDM (Fused Deposition Modeling) con filamento de plástico de ácido poliláctico. Las cuerdas vocales se representaron utilizando dedos de guante. Resultados: El marco laríngeo se modeló de tal manera que las cuerdas vocales encajaran en la glotis. La base y la estructura de soporte fueron realizadas en tablero de fibra de madera sintética de densidad media, al que se fija el marco laríngeo mediante un sistema de imanes. La

\footnotetext{
${ }^{1}$ Hospital Universitário Bettina Ferro de Souza, Belém - PA. *E-mail: amanda_umbelino@hotmail.com
} 
simulación se produce mediante la inyección de material en el interior de las cuerdas vocales, observándose un aumento de su volumen. Conclusión: Se observó un alto grado de fidelidad en la representación anatómica y propiedades táctiles del marco osteocartilaginoso, lo que, además de la alta reproducibilidad y el bajo costo, permite integrar los servicios de residencia en la práctica diaria como una nueva metodología de enseñanza.

Palabras clave: Laringe, Entrenamiento simulado, Otolaríngologia.

\section{INTRODUÇÃO}

Os primeiros procedimentos injetáveis na laringe datam de mais de 100 anos atrás, porém têm sido realizados com maior frequência nos últimos 20 anos, tendo como principais indicações o tratamento da insuficiência glótica causada por paralisia/paresia da prega vocal, presbifonia e sulco vocal além do tratamento da distonia laríngea (MALLUR P e ROSEN C, 2010; DION G e NIELSEN S, 2019). A injeção laringoplástica (IL) é a técnica em que é feita a injeção de certos materiais do interior da prega vocal com o objetivo de obter melhoria do padrão vocal, da deglutição, da proteção da via aérea e da qualidade de vida (VASCONCELOS SJ, 2014).

Aliada ao desenvolvimento tecnológico dos videolaringoscópios flexíveis e o surgimento de diversos materiais injetáveis houve a disseminação das técnicas e a realização cada vez mais frequente em ambiente ambulatorial, com o paciente acordado (DION G e NIELSEN S, 2019). Tratam-se de procedimentos com custo muito inferior quando comparados àqueles sob anestesia geral, porém é necessário conhecimento profundo dos marcos anatômicos para guiar agulha através os tecidos do pescoço até a laringe, o que pode ser tecnicamente desafiador (VERMA S e DAILY S, 2013; AINSWORTH T et al., 2014).

Além dos desafios técnicos, as preocupações crescentes com os erros médicos trouxeram à tona a necessidade de melhores métodos para o treinamento, sendo a simulação de grande valia por permitir um ambiente para aprendizado seguro e sem oferecer riscos para os pacientes (AGHA R e FOWLER A, 2015). A utilização dos simuladores permite ao residente um melhor desenvolvimento de habilidades psicomotoras, tornando possível os erros e a repetição da tarefa quantas vezes forem necessários e propiciando um ganho de confiança e redução na curva de aprendizado. Podem ser realísticos, imersivos e com alto nível de engajamento (AGHA R e FOWLER A, 2015).

Técnicas tradicionais para o ensino de procedimentos invasivos e habilidades cirúrgicas envolvem o uso de materiais biológicos como cadáveres humanos ou animais. Apesar do alto grau de fidelidade na simulação quando são utilizados materiais biológicos, deve-se levar em conta questões como disponibilidade, biossegurança e custos associados ao transporte e armazenamento dos espécimes (CHAN H et al., 2015). Desta forma, o desenvolvimento de metodologias alternativas se impõe.

A impressão 3D, também chamada de manufatura aditiva, tem sem mostrado alternativa eficiente do ponto de vista educacional, por permitir simulação realística para cirurgiões em treinamento. Esta tecnologia tem sido usada na otorrinolaringologia há mais de uma década, sendo aplicada na confecção de moldes para aparelhos de amplificação sonora individual desde 2000. Permite a modelagem de estruturas anatômicas complexas com baixo custo, sendo ideal para a simulação cirúrgica, além de permitir modelagens personalizadas que auxiliam o planejamento pré-operatório (VANKOEVRING K et al., 2016).

Os simuladores de procedimentos laríngeos em sua maior parte têm como objetivo o treino de microcirurgia de laringe e procedimentos de acesso à via aérea, como cricotireoidostomia (MUSHBANI O et al, 2016). Apesar do número reduzido de simuladores com foco no treino da IL, foi demonstrado que o uso de simulação da injeção laríngea aumenta em $78 \%$ confiança dos residentes para a realização do procedimento (CABRERA-MUFFLY $C$ et al., 2015).

Apesar da curva de aprendizado inerente à aquisição de uma nova técnica a prática é importante para tornar o procedimento factível para os residentes. Considerando que os simuladores são ferramentas que podem auxiliar no desenvolvimento de habilidades técnicas em um contexto clínico e a necessidade de criação de novas metodologias para o ensino da IL foi desenvolvido este estudo. Dessa forma, o presente 
estudo tem como objetivos o desenvolvimento de um modelo de simulação de injeção laringoplástica transcervical fabricado com impressão 3D e outros materiais de baixo custo, testando a semelhança do modelo com as propriedades táteis da simulação com o procedimento de IL real e avaliar sua a reprodutibilidade e acessibilidade a todos os serviços de residência em Otorrinolaringologia, propondo uma nova forma de aprendizado.

\section{MÉTODOS}

Trata-se de estudo experimental descritivo realizado no serviço de Otorrinolaringologia do Hospital Bettina Ferro de Souza, vinculado à Universidade Federal do Pará, na cidade de Belém - PA. Devido à natureza do projeto, não foi necessária aprovação do Comitê de Ética em Pesquisa. O projeto foi estruturado em 3 etapas: idealização da pesquisa, estudo sobre a viabilidade do projeto e produção do protótipo de treinamento, totalizando um tempo de aproximadamente 11 meses para chegar ao resultado atual. Foram avaliadas a semelhança anatômica com a laringe humana real levando em consideração os seus marcos anatômicos e propriedades táteis, a resposta normal do tecido ao procedimento, reologia do material injetável e a simulação da técnica de IL.

O modelo consiste em 3 segmentos: 1) arcabouço osteocartilaginoso da laringe; 2) pregas vocais, 3) base e estrutura de apoio do simulador. A modelagem das estruturas osteocartilaginosas da laringe foi feita através dos softwares 3D Max e Z Brush, no formato de desenho tridimensional STL - "stereolitography" - a partir de imagens adquiridas através de radiografia do pescoço nas incidências perfil e anteroposterior de um adulto jovem do sexo masculino processadas em formato DICOM (Digital Imaging and Communications in Medicine). As dimensões do modelo foram de $7,5 \mathrm{~cm}$ de altura, $6 \mathrm{~cm}$ de diâmetro anteroposterior e $5 \mathrm{~cm}$ de largura. A impressão do modelo foi feita com de com impressora 3D do tipo FDM (Fused Deposition Modeling) modelo Ender 3 a partir do filamento plástico ácido poliláctico (PLA) da cor branca (Figura1).

Figura 1 - Arcabouço laríngeo após modelagem em software.

A) Visão frontal; B) Visão posterior; C) Visão em perfil; D) Visão posterolateral.

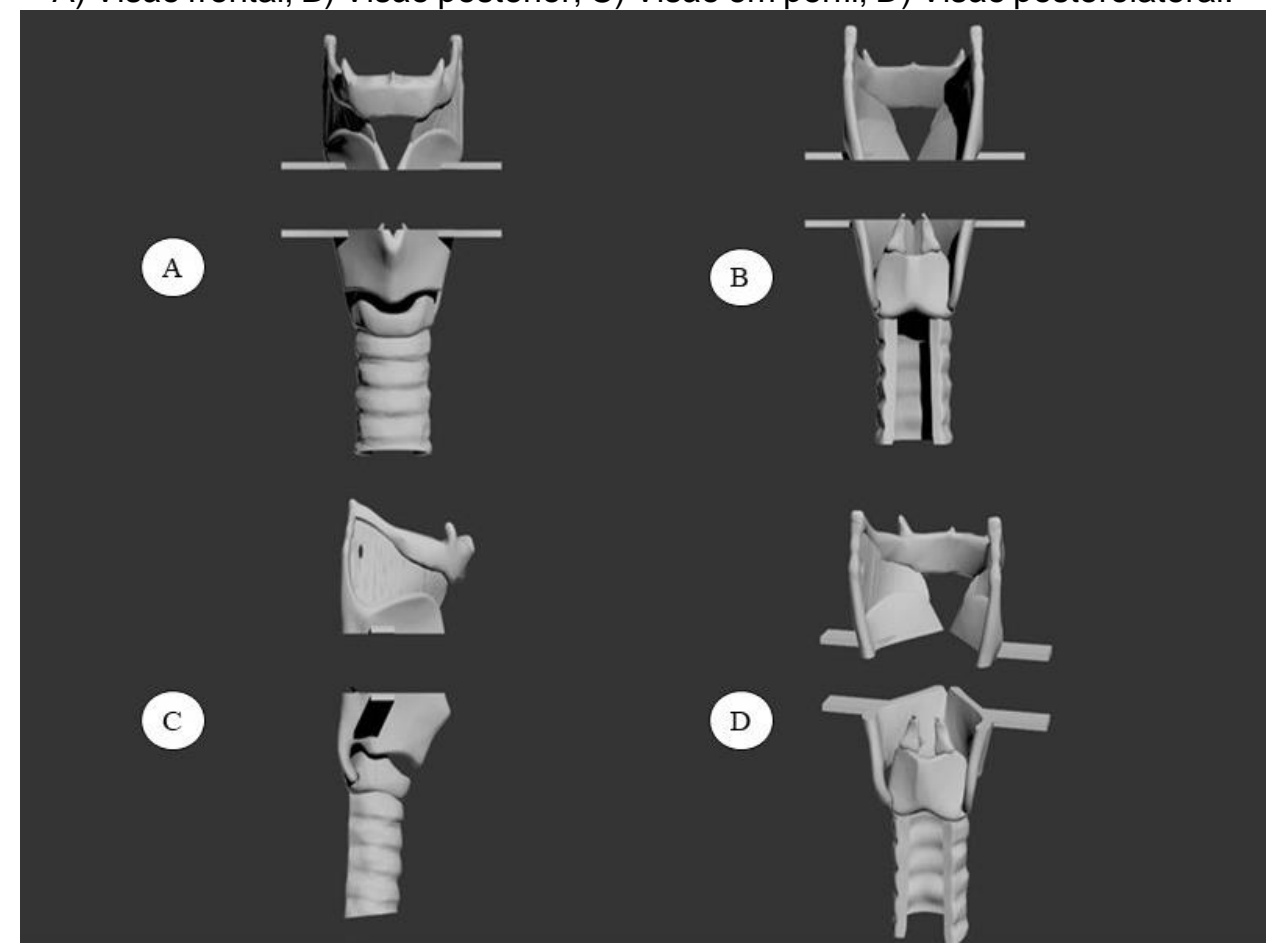

Fonte: Umbelino AM, et al., 2020.

Com o objetivo reduzir o custo e facilitar a produção do modelo, buscou-se materiais de fácil acesso para a sua construção. Desta forma, as pregas vocais foram representadas por dedos de luva de procedimento não cirúrgico a base de látex da marca Descarpack®, amarradas em uma das suas extremidades. 
A simulação foi realizada com a visualização através de sistema vídeo que inclui fibra óptica $3 \mathrm{~mm}$ da marca Xion, angulação de $30^{\circ}$ e $13 \mathrm{~cm}$ de comprimento, fonte de luz Olympus EXERA II CLV-180, câmera Ferrari Medical MFX 10Ge, monitor Radiance NDS H e com microscópio cirúrgico da marca Carl Zeiss OPMI Visu 150 S7, lente de $200 f$ e distância focal de $20 \mathrm{~cm}$.

\section{RESULTADOS}

Foram representadas através da impressão 3D as principais estruturas osteocartilaginosas da laringe: osso hióide, cartilagem tireóidea, cricóide, anéis traqueais e aritenóides. Dessa forma, foi possível a avaliação dos marcos anatômicos utilizados no procedimento real, aumentando assim a fidelidade do simulador. Para que fosse possível manter o arcabouço laríngeo simulando a posição sentada, foi confeccionada base e estrutura de suporte a partir de placa de fibra de madeira sintética de média densidade (medium density fiberboard - MDF) possuindo as dimensões de $20 \mathrm{~cm}$ de comprimento por $20 \mathrm{~cm}$ de largura e $3 \mathrm{~cm}$ de altura. Esta foi construída com dois braços de apoio medindo $8 \mathrm{~cm}$ no sentido vertical que funcionaram como área de fixação do arcabouço através de sistema de ímãs. O arcabouço laríngeo foi modelado de forma a possuir área de encaixe das pregas vocais, ao nível da glote (Figuras 2 e 3).

Figura 2 - Arcabouço laríngeo e estrutura de suporte.

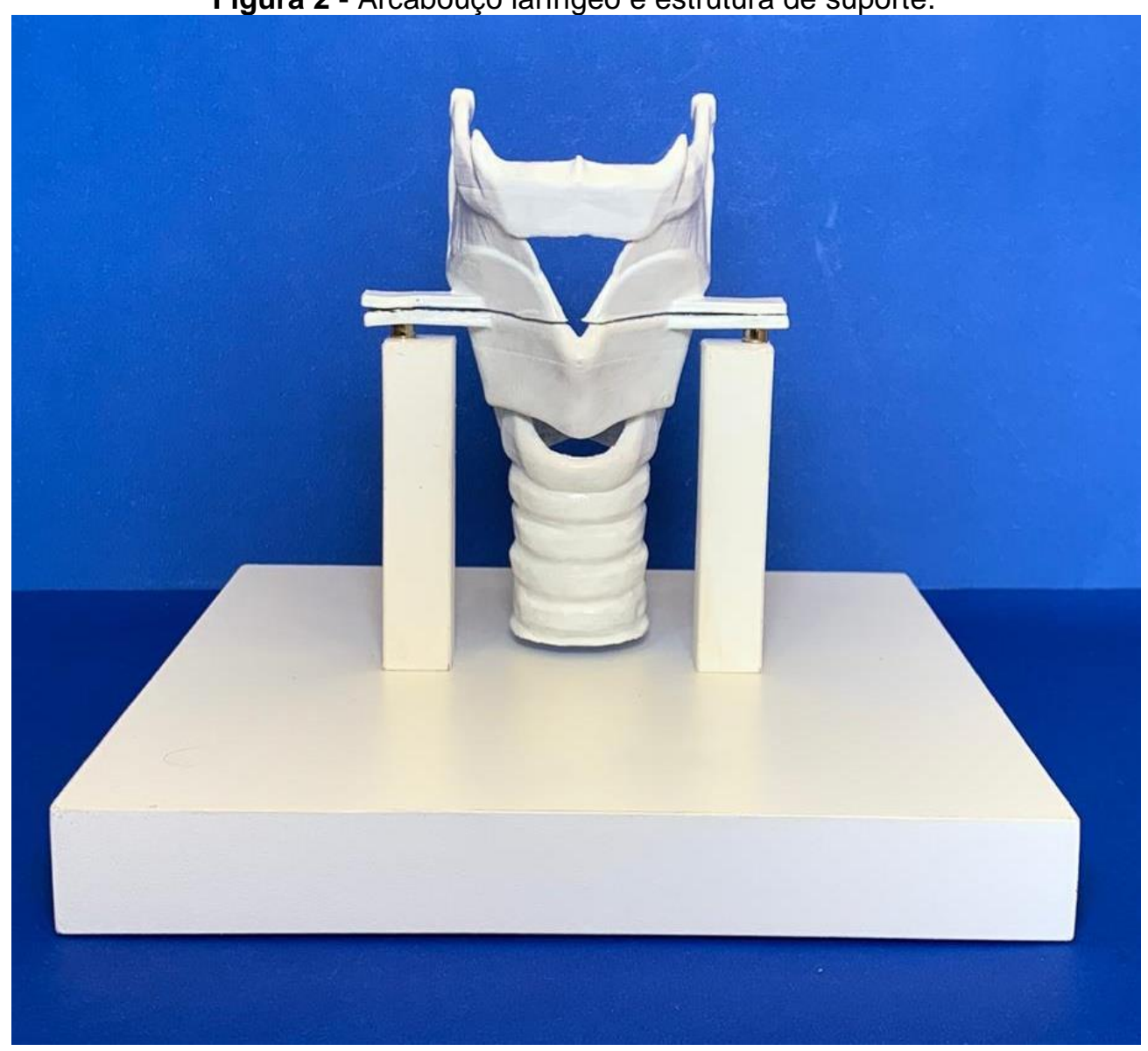

Fonte: Umbelino AM, et al., 2020.

Foram utilizados dois dedos de luva cortados e amarrados em uma de suas extremidades, formando um tubo flexível fechado, sendo obtida assim a vedação necessária para permitir a injeção do material no seu interior sem extravasamento. Devido a flexibilidade proporcionada pelo látex foi possível a injeção do preenchedor sem resistência e sem a ruptura dos dedos de luva. Estes foram presos ao modelo através de um sistema de ímãs presentes nas duas partes do arcabouço laríngeo, proporcionando fixação e certa tensão, mantendo-os esticados, simulando assim, as pregas vocais com volume reduzido e a presença do gap glótico no início da simulação seguido do aumento de volume das pregas vocais e redução do gap após a injeção. 
Como materiais de consumo para o treino da injeção foram utilizados seringa de $5 \mathrm{ml}$ e cateter intravenoso número 20G. Foi realizada a angulação do cateter cerca de 47 graus para permitir melhor acesso às pregas vocais superiormente quando IL realizada pela via transcricotireóidea.

Para IL pela via transtireohidóidea foi utilizada a angulação do cateter para baixo. Com o objetivo de obter as características reológicas ideais para o material injetável aventou-se inicialmente o uso de base para transformação de massas slime incolor à base de polímero polivinílico da marca Altezza Produtos Eireli®, que possui consistência gelatinosa que se acentua ao ser adicionado água boricada $3 \%$ e bicarbonato de sódio.

Ao ser testada a injeção utilizando apenas esta substância, foi observada elevada resistência à passagem pela agulha, sendo necessária aplicação de força desproporcional à necessária no procedimento real. Para tornar a consistência do material mais fluida este material foi combinado com água na proporção de 1:1. O custo total do simulador foi de $\mathrm{R} \$ 170,00$.

Figura 3 - A) visão superior do arcabouço laríngeo, sem o encaixe das pregas vocais; B) Visão do sistema de ímãs para encaixe das pregas vocais; C) visão superior das pregas vocais presas através do sistema de ímãs, notar volume reduzido; D) material de consumo utilizado para o treino da injeção: dedos de luva, seringa de $5 \mathrm{ml}$, cateter intravenoso número 20G e material polivinílico

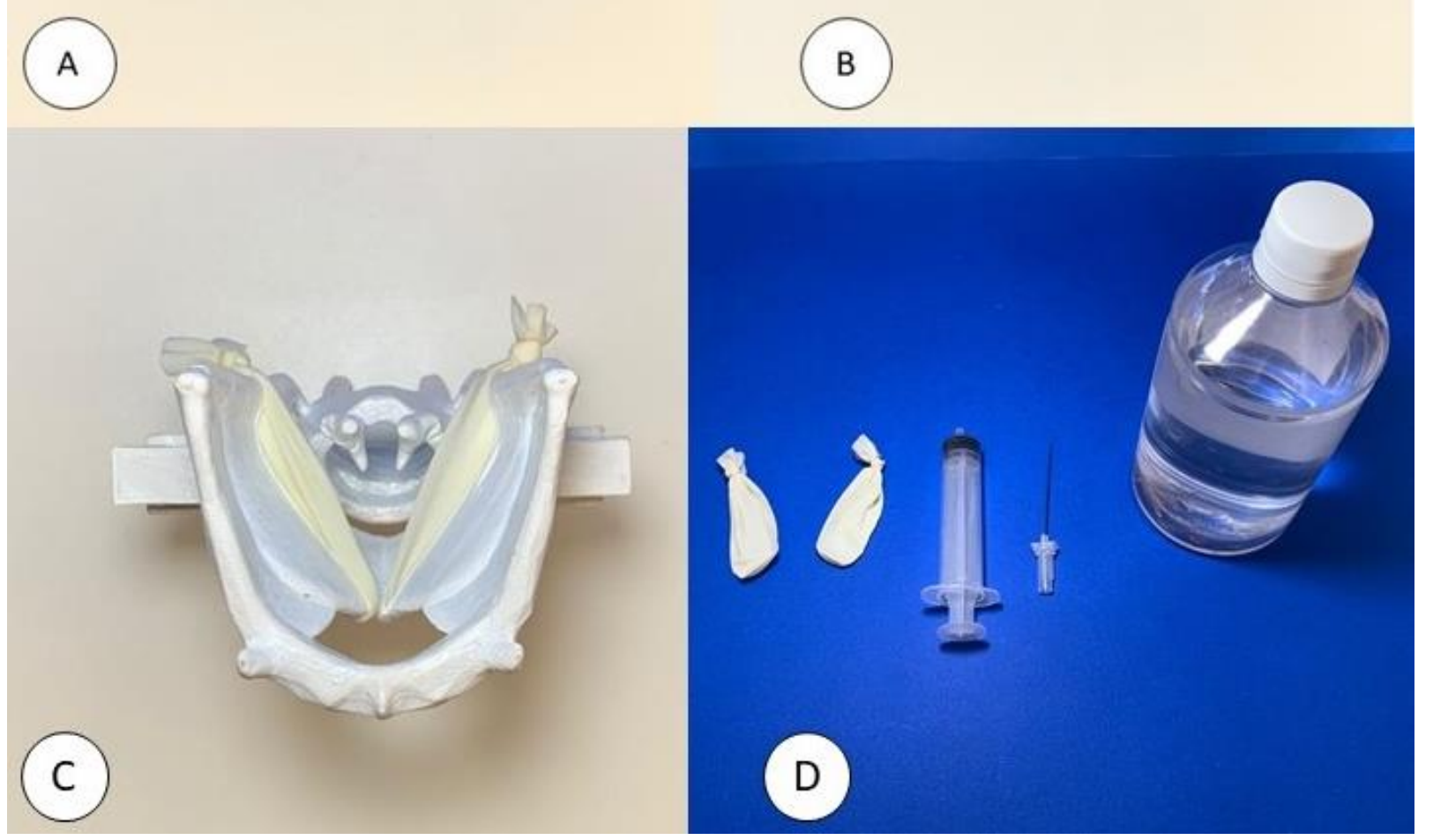

Fonte: Umbelino AM, et al., 2020. 
A simulação do procedimento se dá com o modelo em posição vertical, mimetizando o paciente sentado, sendo feita a visualização das pregas vocais através de sistema de vídeo posicionado superiormente ao simulador com a ajuda de um auxiliar ou sob visão microscópica (Figura 4).

Figura 4 - Realização da simulação da IL por via transtireohiódea com visualização por sistema de vídeo com fibra ótica rígida posicionada superiormente ao modelo.

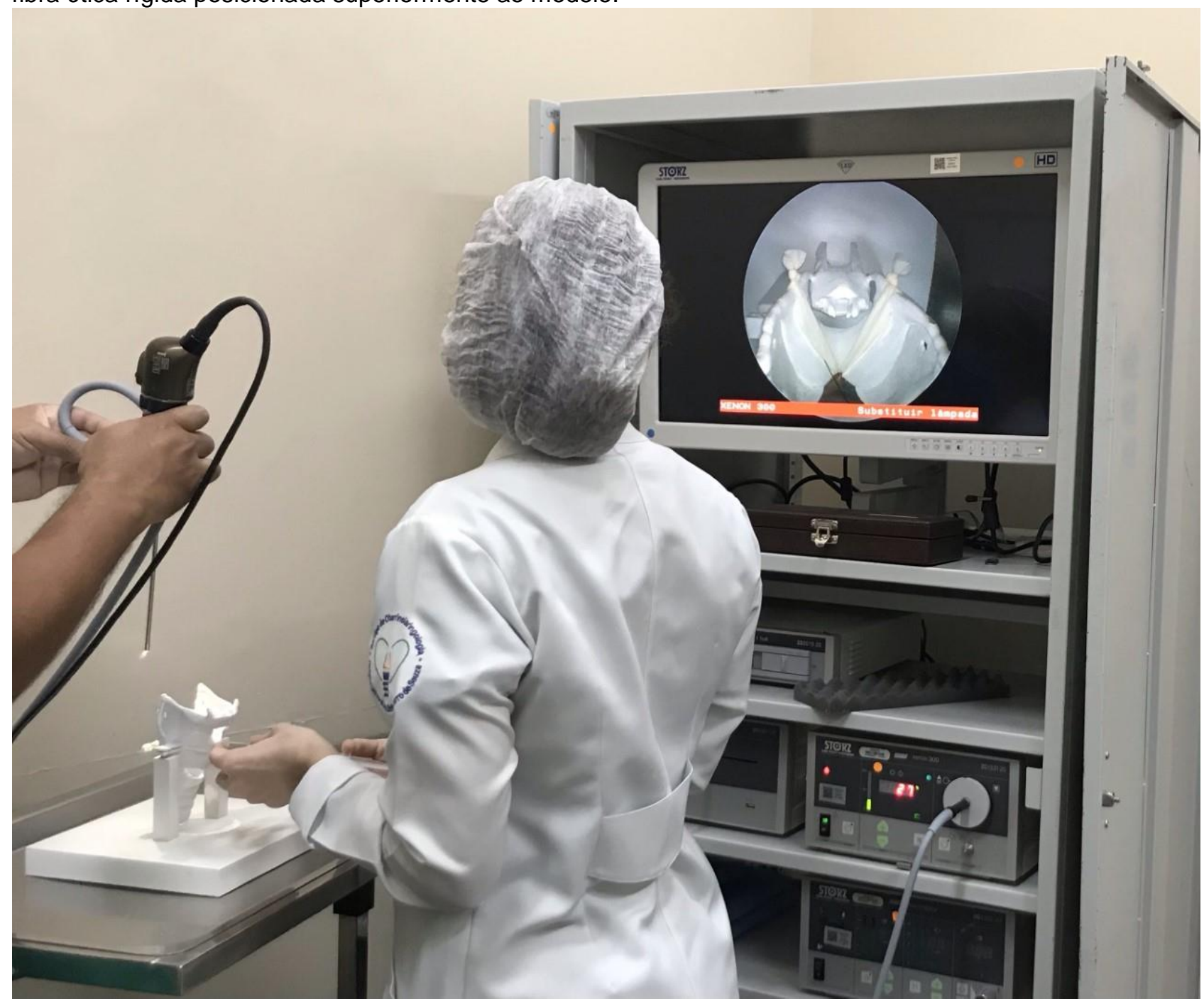

Fonte: Umbelino AM, et al., 2020.

É feita a visualização das pregas vocais com volume reduzido e observada a presença do gap glótico, sendo orientada então a identificação dos pontos anatômicos de referência: cartilagem tireóide, região cricotireóidea, cartilagem cricóide e proeminência tireóidea.

A agulha é inserida na margem inferior da cartilagem tireóide, cerca de $7 \mathrm{~mm}$ lateral à linha média através da região cricotireóidea, devendo seguir até a região das pregas vocais. Ao nível destas, é visualizada a ponta da agulha por transparência no interior da prega vocal, sendo confirmada a inserção no local desejado, sendo feita assim a injeção do material.

Para a simulação da IL pela via transtireohióide a agulha é guiada através da região imediatamente superior à cartilagem tireóide, até as pregas vocais inferiormente, sendo possível a visualização direta da inserção da agulha no interior da prega vocal. A simulação é finalizada com a observação do aumento de volume e medialização da prega vocal após a injeção do material, sendo obtido o resultado desejado de redução do gap glótico (Figura 5). 
Figura 5 - Resultado final da simulação após a injeção do preenchedor: A) realizada IL apenas em prega vocal direita, notar diferença de volume entre as pregas vocais; B) após realização da IL em ambas as pregas vocais, notar redução do gap glótico

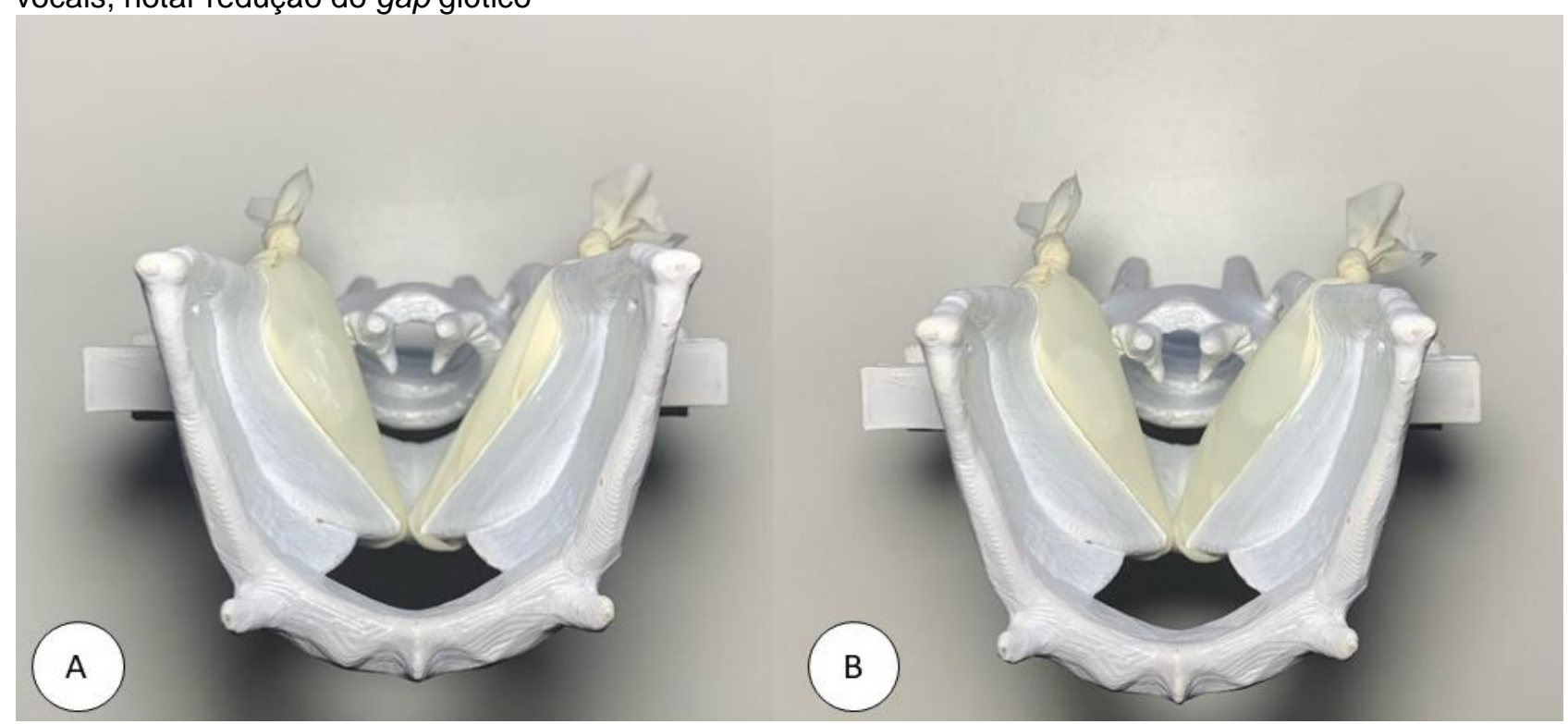

Fonte: Umbelino AM, et al., 2020.

\section{DISCUSSÃO}

A Otorrinolaringologia é especialidade clínico-cirúrgica que tem como características procedimentos desafiadores, muitas vezes com um campo cirúrgico reduzido em um sítio anatômico com proximidade de várias estruturas nobres, que é a região da cabeça e pescoço. Com a necessidade de melhorar a eficiência no aprendizado das habilidades cirúrgicas na especialidade um número crescente de simuladores vem sendo desenvolvido. De acordo com revisão sistemática realizada por Mushbani O, et al. (2016) estão descritos na literatura 64 modelos de simuladores em otorrinolaringologia, sendo 32 de procedimentos otológicos, 20 de laringe e faringe e 12 de cirurgia endoscópica nasal. Destes, apensas quatro são modelos de IL.

Desde da introdução da técnica de IL através da injeção transoral com laringoscopia indireta por Bruenings em 1911 esta tem se consolidado no tratamento da insuficiência glótica. O desenvolvimento tecnológico dos videolaringoscópios, dos diferentes materiais injetáveis e das técnicas de anestesia permitiram a reemergência do procedimento, sendo este o procedimento ambulatorial mais realizado por laringologistas (BENSOUSSAN Y e ANDERSON J, 2018). A IL comumente é realizada em ambiente ambulatorial, com o paciente acordado, tornando mais difícil o ensino da técnica aos residentes e a simulação oferece a oportunidade de treinamento em um ambiente seguro, sem riscos ao paciente e permitindo o erro.

O uso da impressão 3D, dentre os materiais sintéticos, possui a vantagem de permitir a representação anatômica com alta fidelidade e baixo custo. Neste estudo observamos alto grau de fidelidade na representação anatômica e das propriedades táteis do arcabouço osteocartilaginoso da laringe através da impressão 3D, sendo possível a reprodução em detalhes das estruturas, respeitando inclusive acidentes ósseos e cartilaginosos no tamanho real da laringe de um adulto do sexo masculino. Isto possibilita, durante a simulação, a palpação de marcos anatômicos de referência, avaliação do sítio adequado de introdução da agulha, o treino da angulação adequada desta e da distância que deverá ser percorrida até o local da injeção, aumentando muito a fidelidade em relação ao procedimento no paciente real.

A modelagem do arcabouço laríngeo em software de impressão 3D possibilita a reimpressão deste quantas vezes forem necessárias, tornando possível a produção em maior escala, caso desejado. A acessibilidade do modelo também é grande uma vez que foi utilizado a impressão 3D modelo FDM, que possui maior disponibilidade e menor custo, no valor total de $\mathrm{R} \$ 150,00$. Uma vez que se trata de um material durável, permite a repetição da simulação quantas vezes forem necessárias após simples troca dos dedos de luva e limpeza de eventuais resíduos presentes em sua estrutura. 
No que se refere à utilização dos dedos de luva observou-se boa representação do objetivo final do procedimento no sentido em que foi possível a visualização do aumento do volume destes, confirmando o adequado posicionamento da agulha e a resposta do tecido normal ao preenchimento com material injetável. Com o material injetável escolhido para a simulação foi possível obter viscosidade ideal, coesão, fluidez adequada na agulha e resistência ao extravasamento na prega vocal através da região de penetração da agulha. Foram necessários cerca de $1 \mathrm{ml}$ em cada prega vocal para obter o resultado esperado.

Apesar da simulação poder ser realizada sem nenhum método de amplificação da imagem, através da visualização direta na extremidade superior do modelo, a utilização da videolaringoscopia pode proporcionar oportunidade para aquisição de habilidades necessárias aos procedimentos por vídeo, como a noção de profundidade e melhora a coordenação entre os movimentos da mão e os movimentos observados pelos olhos, que costumam ser desafiadores para os residentes. Considerando que a IL ambulatorial é realizada com o auxílio desta tecnologia os autores consideraram importante a sua inclusão na simulação.

Outros simuladores de injeção laríngea descritos na literatura incluem dois modelos de material biológico e três de material sintético. Os modelos biológicos desenvolvidos por Amin M, et al. (2007), Awad Z, et al. (2014) e Cha W, et al. (2018) foram feitos com cadáver humano fresco, laringe porcina e laringe canina respectivamente. O modelo de Amin $\mathrm{M}$, et al. (2007) contou com uma laringe humana fresca, apoiada em cabeça de manequim de intubação para treino da IL via transoral com visualização por videolaringoscopia. $O$ nível de desconforto relatado pelos residentes com o procedimento foi de 22 pré simulação para 52 pós simulação $(0=$ nem um pouco confortável e 100= muito confortável). Awad $Z$, et al. (2014) utilizou a laringe porcina para treino de diferentes procedimentos além da injeção laríngea como coleta de biópsia de prega vocal e elevação de flap submucoso sendo validado através de questionário por mais de $75 \%$ dos participantes.

Cha W, et al. (2018) utilizou laringe canina fixada em suporte tubular e agulhas conectadas à uma fonte de luz através de cabo de fibra óptica, com visualização da injeção através de videolaringoscopia com o objetivo de identificar a localização da agulha durante o procedimento por via transcricotireóidea. Foi possível a adequada identificação da ponta da agulha em diferentes pontos de injeção e não foi desenvolvido questionário de validação. Apesar de certamente apresentarem um alto grau de fidelidade ao procedimento real estes simuladores possuem as limitações associadas ao alto custo, risco de contaminação biológica e baixa disponibilidade o que dificulta a aplicação rotineira nos serviços públicos de residência. Além disso, o modelo descrito neste estudo, por ser totalmente sintético proporciona a facilidade de poder ser utilizado mesmo fora do ambiente hospitalar.

O modelo sintético desenvolvido por Ainsworth T, et al. (2014) foi o primeiro a utilizar feedback auditivo em tempo real, sendo desenvolvida a estrutura laríngea através de impressão 3D e inseridos filamentos metálicos no músculo tireoaritenódeo. Estes filamentos foram ligados à cabos elétricos conectados a um microfone e à uma caixa de som, que emitiam feedback auditivo ao contato com eletrodo presente na agulha da injeção, confirmando a localização correta da agulha. Foi relatado pelos residentes melhora da confiança para a realização do procedimento após a aplicação do modelo de 1.2 para 2.9 para residentes do primeiro ano e de 3.4 para 5.6 para residentes do segundo ano $(1=$ desconfortável e $10=$ muito confortável). Não foi informado o custo envolvido na manufatura do modelo, porém certamente possui um custo mais elevado e maior complexidade na confecção em comparação ao modelo proposto por depender de material elétrico especializado, o que pode dificultar a produção em maior escala. Até o momento, o simulador descrito no presente estudo é o único para treino de IL de baixo custo produzido através da impressão 3D.

O segundo modelo sintético, criado por Cabrera-Muffly C, et al. (2015) consistiu de um simulador de baixo custo criado com rolo de papel higiênico, papel cartão, fixador plástico de cabos, balão e tecido. Com estes materiais foram simulados o arcabouço laríngeo, cartilagem tireóide, cricóide e pregas vocais, sendo orientado a palpação dos pontos de referência anatômica, injeção de material e observação do aumento de volume das pregas vocais. Também foi relatado melhora na confiança para a realização do procedimento após a aplicação da simulação por $78 \%$ dos residentes. Este modelo possuiu a vantagem do custo bastante reduzido e do uso de materiais de fácil acesso, no entanto apresenta baixa fidelidade ao procedimento real. Não foi encontrado na literatura nenhum simulador de procedimento laríngeo de realidade virtual. 
No presente estudo foi possível a criação de um modelo sintético para treinamento de IL com materiais de baixo custo, com boa reprodutibilidade, de baixo peso e de fácil transporte e com a possibilidade de uso repetidas vezes o que torna possível a integração na prática diária dos serviços de residência como nova metodologia de ensino. Apresenta muitas diferenças em comparação aos modelos biológicos classicamente utilizados que além de necessitarem de cuidados de biossegurança apresentam baixa disponibilidade e alto custo. Em comparação aos modelos sintéticos descritos na literatura podemos destacar a possibilidade da representação das estruturas anatômicas da laringe com certo grau de fidelidade através da impressão 3D aliada à utilização de materiais de baixo custo e altamente acessíveis, que podem ser confeccionados por qualquer pessoa. Este fato é extremamente importante levando em consideração o objetivo de propor uma metodologia que seja aplicável à realidade dos hospitais universitários.

Um aspecto não satisfatório e que precisa ser alvo de adaptações foi impossibilidade de representar adequadamente as partes moles e estruturas membranáceas e musculares da laringe através da impressão 3D. Isto está relacionado ao fato de ser utilizado impressora FDM com o filamento PLA, material que caracteristicamente possui maior rigidez. Esta limitação pode ser sanada através do uso de impressoras multimaterial ou da aplicação de outros materais de baixo custo que possam simular os ligamentos, membranas e tecidos moles adjacentes.

Achado semelhante foi observado por Kavanagh K, et al. (2016) que compararam a rigidez e a facilidade de manipulação de um simulador de cirurgia em via aérea pediátrica feito de diferentes filamentos de impressão 3D e de elastômero de silicone produzido a partir de molde bipartido confeccionado em impressora 3D. Os autores observaram elevada rigidez nos modelos feitos puramente em impressão 3D e obtiveram um modelo passível de manipulação cirúrgica através de incisões, dilatações e até sutura no modelo feito a partir de elastômero de silicone. Uma alternativa para aliar a fidelidade anatômica à fidelidade tátil pode ser a utilização do molde impresso em 3D para confecção de modelos à base de silicone. A melhora deste aspecto irá possibilitar a possibilidade de palpação da membrana cricotireóidea, importante marco anatômico para a realização do procedimento, além de permitir também a realização da IL por via transtireóidea.

\section{CONCLUSÃO}

Neste estudo foi descrito um modelo de simulador feito com impressão 3D e outros materiais de baixo custo de fácil aplicação, que permite a identificação dos parâmetros anatômicos com boa fidelidade ao procedimento real e que pode facilmente ser incorporado aos serviços de residência, sem as limitações relacionadas aos tradicionais simuladores de material biológico como disponibilidade, biossegurança, questões éticas e custos relacionados ao armazenamento e transporte dos espécimes. Além disso, pelo fato de ser possível a troca da estrutura correspondente às pregas vocais o simulador pode ser utilizado repetidas vezes sem prejuízo da qualidade do modelo. O estudo teve limitações, principalmente a impossibilidade de representar adequadamente as partes moles e estruturas membranáceas e musculares da laringe através da impressão 3D devido ao uso de filamento com alta rigidez. Outra limitação foi a ausência de aplicação de protocolo de validação do modelo. Como sugestões para estudos futuros propomos a confecção do simulador em impressora multimaterial para melhor representação das partes moles laríngeas e para treino de todas as vias de IL. Sugerimos também, após as devidas adaptações a validação por parte dos residentes e otorrinolaringologistas com o objetivo de quantificar as habilidades adquiridas.

\section{REFERÊNCIAS}

1. AGHA R, FOWLER A. The Role and Validity of Surgical Simulation. International Surgery, 2015

2. AINSWORTH T. et al. Simulation Model for Transcervical Laryngeal Injection Providing Real-time Feedback. Annals of Otology, Rhinology \& Laryngology 2014, Vol. 123

3. AMIN M, et al. Hands-On Training Methods for Vocal Fold Injection Education. Annals of Otology, Rhinology \& Laryngology 116(1):1-6, 2007

4. AWAD Z, et al. Simulation in laryngology training; what should we invest in? Our experience with 64 porcine larynges and a literature review. Clinical Otolaryngology, 2014.

5. BENSOUSSAN Y, ANDERSON J. In-office laryngeal procedures (IOLP) in Canada: current safety practices and procedural care. Journal of Otolaryngology - Head and Neck Surgery, 2018 
6. BARBALHO C. Avaliação clínica, motora e laringoscópica de pacientes com doença do refluxo gastroesofágico e exposição ácida patológica, com e sem sintomas laringofaríngeos. Fortaleza, 2019. Dissertação de Mestrado (Mestrado em Cirurgia). Programa de Pós-graduação em Cirurgia da Faculdade de Medicina da Universidade Federal do Ceará, 2019

7. BENSOUSSAN Y, ANDERSON J. In-office laryngeal procedures (IOLP) in Canada: current safety practices and procedural care. Journal of Otolaryngology - Head and Neck Surgery, 2018

8. CHA W, et al. Real-Time Light-Guided Vocal Fold Injection: Ex Vivo Feasibility Study in a Canine Model. The Laryngoscope. January, 2018.

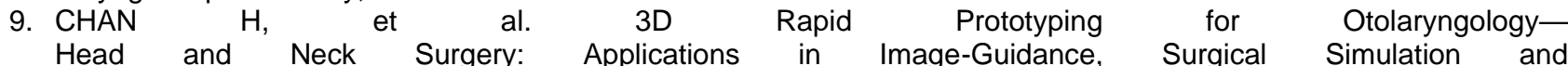
Patient-Specific Modeling. PLOS ONE September 2, $2015 \quad$ Surgical Simulation and

10. CHANDRAN D, et al. A comparative study of voice outcomes and complication rates in patients undergoing injection laryngoplasty performed under local versus general anaesthesia: an Adelaide voice specialist's experience. The Journal of Laryngology \& Otology. Vol 131, 2017

11. CABRERA-MUFFLY C, et al. A Low-Cost Transcervical Laryngeal Injection Trainer. The Laryngoscope. Abril, 2015

12. DEFATTA R, et al. Complications of Injection Laryngoplasty Using Calcium Hydroxylapatite. Journal of Voice, Vol. 26, No. 5, 2012

13. DION G, NIELSEN S. In-Office Laryngology Injections. Otolaryngolic Clinics of North America, 2019

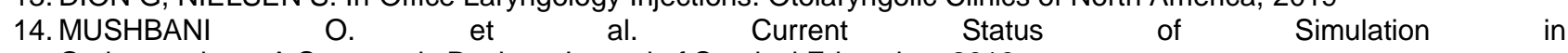
Otolaryngology: A Systematic Review. Journal of Surgical Education, 2016

15. MALLUR P, ROSEN C. Vocal Fold Injection: Review of Indications, Techniques, and Materials for Augmentation. Clinical and Experimental Otorhinolaryngology Vol. 3, No. 4: 177-182, December 2010

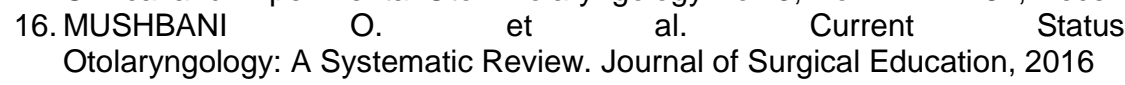

17. KAVANAGH K, et al. Pediatric Laryngeal Simulator Using 3D Printed Models: A Novel Technique. The Laryngoscope 00: Month, 2016

18. VASCONCELOS SJ. Efeito da injeção de biopolímero da cana-de-açúcar na prega vocal de coelhos: estudo comparativo com a hidroxiapatita de cálcio. São Paulo, 2014. 84 f. Tese (Doutorado em Ciências) - Programa de Otorrinolaringologia. Faculdade de Medicina da Universidade de São Paulo. 2014

19. VERMA S, DAILEY S. Office-Based Injection Laryngoplasty for the Management of Unilateral Vocal Fold Paralysis. Journal of Voice, Vol. 28, No. 3, 2014

20. VANKOEVERING K, MALLOY K. Emerging Role Three-Dimensional Printing in Simulation in Otolaryngology. Otolaryngolic Clinics of North America 50 (2017) $947-958$. 